Article

\title{
Generalized Quasi-Einstein Manifolds in Contact Geometry
}

\author{
İnan Ünal
}

Department of Computer Engineering, Munzur University, 62000 Tunceli, Turkey; inanunal@munzur.edu.tr; Tel.: +90-428-213-1794

Received: 17 July 2020; Accepted: 31 August 2020; Published: 16 September 2020

\begin{abstract}
In this study, we investigate generalized quasi-Einstein normal metric contact pair manifolds. Initially, we deal with the elementary properties and existence of generalized quasi-Einstein normal metric contact pair manifolds. Later, we explore the generalized quasi-constant curvature of normal metric contact pair manifolds. It is proved that a normal metric contact pair manifold with generalized quasi-constant curvature is a generalized quasi-Einstein manifold. Normal metric contact pair manifolds satisfying cyclic parallel Ricci tensor and the Codazzi type of Ricci tensor are considered, and further prove that a generalized quasi-Einstein normal metric contact pair manifold does not satisfy Codazzi type of Ricci tensor. Finally, we characterize normal metric contact pair manifolds satisfying certain curvature conditions related to $\mathcal{M}$-projective, conformal, and concircular curvature tensors. We show that a normal metric contact pair manifold with generalized quasi-constant curvature is locally isometric to the Hopf manifold $S^{2 n+1}(1) \times S^{1}$.
\end{abstract}

Keywords: generalized quasi-Einstein; contact pairs; generalized quasi-constant curvature; $\mathcal{M}$-projective curvature tensor

\section{Introduction}

An Einstein manifold is a Riemannian manifold $(M, g)$, which is defined by the Ricci tensor Ric $=\lambda g$ for a non-zero constant $\lambda$. Since Einstein manifolds have important differential geometric properties and have significant physical applications therefore they are studied by geometers in a broad perspective. A Riemannian manifold $M$ is called a quasi-Einstein manifold if the Ricci curvature tensor satisfies

$$
\operatorname{Ric}\left(X_{1}, X_{2}\right)=\lambda g\left(X_{1}, X_{2}\right)+\beta \omega\left(X_{1}\right) \omega\left(X_{2}\right)
$$

for all $X_{1}, X_{2} \in \Gamma(T M)$, where $\lambda, \beta$ are scalars and $\omega$ is a non-zero 1 -form [1]. Quasi-Einstein manifolds are generalizations of Einstein manifolds. In the contact geometry, $\eta$-Einstein manifolds can be consider as a particular case of quasi-Einstein manifolds. When quasi-umbilical hypersurfaces were considered exact solutions of the Einstein field equations, the notion of quasi-Einstein manifold aroused [2]. As an example of quasi-Einstein manifolds, we can mention the Robertson-Walker space-times [2]. For more details on such manifolds, we refer to the reader [2-4].

The generalization of quasi-Einstein manifolds has been presented in the different perspectives. Chaki gave one of them in [5], and another was presented by Catino [6]. Catino generalized a quasi-Einstein manifold as a generalization of the concepts of Ricci solitons and quasi-Einstein manifolds. The third definition of generalized quasi-Einstein manifolds was given by De and Ghosh [7]. A Riemannian manifold $(M, g)$ is called a generalized quasi-Einstein manifold if its Ricci tensor has following form:

$$
\operatorname{Ric}\left(X_{1}, X_{2}\right)=\lambda g\left(X_{1}, X_{2}\right)+\beta \omega\left(X_{1}\right) \omega\left(X_{2}\right)+\mu \eta\left(X_{1}\right) \eta\left(X_{2}\right)
$$


where $\omega, \eta$ are two non-zero $1-$ forms and $\lambda, \beta, \mu$ are certain non-zero scalars [7]. The unit and orthogonal vector fields $\xi_{1}$ and $\xi_{2}$ corresponding to the 1 -forms $\omega$ and $\eta$ are defined by $g\left(X_{1}, \xi_{1}\right)=$ $\omega\left(X_{1}\right), g\left(X_{2}, \xi_{2}\right)=\eta\left(X_{1}\right)$, respectively [7]. The geometric properties of generalized quasi-Einstein manifolds have been studied in [7-11]. A generalized quasi-Einstein manifold, in addition to its geometrical features, has remarkable physical applications in general relativity [12-14]. Complex $\eta$-Einstein manifolds could be considered as a special case of generalized quasi-Einstein manifolds (see [15]).

In [16], Bande and Hadjar defined a new contact structure on an $(m=2 p+2 q+2)$-dimensional differentiable manifold $M$ with two 1 -forms $\alpha_{1}, \alpha_{2}$. This structure was initially studied by Blair, Ludden and Yano [17] as the name of bicontact manifolds. Bande and Hadjar considered a special type of $f$-structure with complementary frames related to these contact forms and they obtained associated metric. A differentiable manifold with this structure is called a metric contact pair (MCP) manifold. Riemannian geometry of MCP manifolds is given in [18,19].

This paper is on applications of generalized quasi-Einstein manifolds in contact geometry. We consider the generalized quasi-Einstein normal metric contact pair manifolds. After presenting definitions and basic properties, we examine the existence of such manifolds. Also, we present a characterization of generalized quasi-Einstein normal metric contact pair manifolds. Moreover, we consider the notion of generalized quasi-constant curvature for normal metric contact pair manifolds and we obtain some results on the sectional curvature. We investigate a generalized quasi-Einstein normal metric contact pair manifold under some conditions for Ricci tensor. We prove that a generalized quasi-Einstein normal metric contact pair manifold does not satisfy Codazzi type of Ricci tensor. Finally, we characterize normal metric contact pair manifolds satisfying certain curvature conditions related to $\mathcal{M}$-projective, conformal, and concircular curvature tensors. We show that a normal metric contact pair manifold with generalized quasi-constant curvature is locally isometric to the Hopf manifold $S^{2 n+1}(1) \times S^{1}$.

\section{Preliminaries}

Contact pairs were defined by Bande and Hadjar [16] in 2005, for details see $[16,18,19]$. In this section, we give some fundamental facts about contact metric pair manifolds. Also, we present some general facts and results on generalized quasi-Einstein manifolds.

Definition 1. Let $M$ be an $(m=2 p+2 q+2)$-dimensional differentiable manifold and $\alpha_{1}, \alpha_{2}$ be two 1-forms on $M$. If the following properties are satisfied then, the pair of $\left(\alpha_{1}, \alpha_{2}\right)$ is called a contact pair of type $(p, q)$ on $M$ :

- $\quad \alpha_{1} \wedge\left(d \alpha_{1}\right)^{p} \wedge \alpha_{2} \wedge\left(d \alpha_{2}\right)^{q} \neq 0$,

- $\left(d \alpha_{1}\right)^{p+1}=0$ and $\left(d \alpha_{2}\right)^{q+1}=0$,

where $p, q$ are positive integers. Then, $\left(M, \alpha_{1}, \alpha_{2}\right)$ is known as a contact pair manifold [16].

The kernels of 1 -forms $\alpha_{1}$ and $\alpha_{2}$ define two subbundles of $T M$ as $\mathcal{D}_{1}=\left\{X: \alpha_{1}(X)=0, X \in\right.$ $\Gamma(T M)\}$ and $\mathcal{D}_{2}=\left\{X: \alpha_{2}(X)=0, X \in \Gamma(T M)\right\}$. Also, we have two characteristic foliations of $M$, denoted by $\mathcal{F}_{1}=\mathcal{D}_{1} \cap \operatorname{kerd} \alpha_{1}$ and $\mathcal{F}_{2}=\mathcal{D}_{2} \cap$ kerd $\alpha_{2}$ respectively. $\mathcal{F}_{1}$ and $\mathcal{F}_{2}$ are completely integrable and their leaves are equipped with a contact form induced by $\alpha_{2}$ (respectively, $\alpha_{1}$ ). On the other hand, the tangent bundle of $M$ can be split as $T M=T \mathcal{F}_{1} \oplus T \mathcal{F}_{2}$ [19]. The horizontal sub-bundle $\mathcal{H}$ of $T M$ can be defined as $\mathcal{H}=k e r \alpha_{1} \cap k e r \alpha_{2}$.

In the contact geometry, we have the characteristic vector field associated with the contact form. Similarly, for a contact pair $\left(\alpha_{1}, \alpha_{2}\right)$ of type $(p, q)$ we have two vector fields $Z_{1}$ and $Z_{2}$, which are uniquely determined by the following equations:

$$
\begin{gathered}
\alpha_{1}\left(Z_{1}\right)=\alpha_{2}\left(Z_{2}\right)=1, \alpha_{1}\left(Z_{2}\right)=\alpha_{2}\left(Z_{1}\right)=0, \\
i_{Z_{1}} d \alpha_{1}=i_{Z_{1}} d \alpha_{2}=i_{Z_{2}} d \alpha_{2}=0
\end{gathered}
$$


where $i_{X}$ is the contraction with the vector field X [16].

Two sub-bundles of TM are defined as follows

$$
T \mathcal{G}_{i}=\operatorname{kerd} \alpha_{i} \cap \operatorname{ker} \alpha_{1} \cap \operatorname{ker} \alpha_{2}, i=1,2 .
$$

Then, we have

$$
T \mathcal{F}_{1}=T \mathcal{G}_{1} \oplus \mathbb{R} Z_{2} \text {, and } T \mathcal{F}_{2}=T \mathcal{G}_{2} \oplus \mathbb{R} Z_{1} .
$$

Therefore, we get $T M=T \mathcal{G}_{1} \oplus T \mathcal{G}_{2} \oplus \mathbb{R} Z_{1} \oplus \mathbb{R} Z_{2}$. The horizontal sub-bundle can be written as $\mathcal{H}=T \mathcal{G}_{1} \oplus T \mathcal{G}_{2}$. Also, we write $\mathcal{V}=\mathbb{R} Z_{1} \oplus \mathbb{R} Z_{2}$, and $\mathcal{V}$ is called the vertical sub-bundle of $T M$. Consequently, the tangent bundle of $M$ is given by $T M=\mathcal{H} \oplus \mathcal{V}$ [18].

Let $X$ be an arbitrary vector field on $M$. We can write $X=X^{\mathcal{H}}+X^{\mathcal{V}}$, where $X^{\mathcal{H}}, X^{\mathcal{V}}$ horizontal and vertical component of $X$, respectively. We call a vector field $X$ as a horizontal vector field if $X \in \Gamma(\mathcal{H})$, and a vertical vector field if $X \in \Gamma(\mathcal{V})$.

Similar to almost complex structures, in the 1960s, almost contact structures were defined with tensorial viewpoint [20]. On a contact pair manifold $M$, an almost contact pair structure has been defined as follow by Bande and Hadjar [18].

Definition 2. An almost contact pair structure on an $(m=2 p+2 q+2)$-dimensional differentiable manifold $M$ is a triple $\alpha_{1}, \alpha_{2}, \phi$, where $\left(\alpha_{1}, \alpha_{2}\right)$ is a contact pair and $\phi$ is a $(1,1)$ tensor field such that:

$$
\phi^{2}=-I+\alpha_{1} \otimes Z_{1}+\alpha_{2} \otimes Z_{2}, \phi Z_{1}=\phi Z_{2}=0 .
$$

The rank of $\phi$ is $(2 p+2 q)$ and $\alpha_{1}(\phi)=\alpha_{2}(\phi)=0[18]$.

Definition 3. $\phi$ is known as decomposable i.e., $\phi=\phi_{1}+\phi_{2}$, if $T \mathcal{F}_{i}$ is invariant under $\phi$ [18].

If $\phi$ is decomposable, then $\left(\alpha_{i}, Z_{i}, \phi\right)$ induces an almost contact structure on $\mathcal{F}_{j}$ for $i \neq j, i, j=1,2$ [16]. The decomposability of $\phi$ does not satisfy for every almost contact pair structure. An example was given in [16], which has an almost contact pair structure, but $\phi$ is not decomposable. In this study, we assume that $\phi$ is decomposable.

Definition 4. Let $\left(\alpha_{1}, \alpha_{2}, Z_{1}, Z_{2}, \phi\right)$ be an almost contact pair structure on a Riemannian manifold $(M, g)$. The Riemannian metric $g$ is called [18]

- compatible if $g\left(\phi X_{1}, \phi X_{2}\right)=g\left(X_{1}, X_{2}\right)-\alpha_{1}\left(X_{1}\right) \alpha_{1}\left(X_{2}\right)-\alpha_{2}\left(X_{1}\right) \alpha_{2}\left(X_{2}\right)$ for all $X_{1}, X_{2} \in T M$,

- associated if $g\left(X_{1}, \phi X_{2}\right)=\left(d \alpha_{1}+d \alpha_{2}\right)\left(X_{1}, X_{2}\right)$ and $g\left(X_{1}, Z_{i}\right)=\alpha_{i}\left(X_{1}\right)$, for $i=1,2$ and for all $X_{1}, X_{2} \in \Gamma(T M)$.

4-tuple $\left(\alpha_{1}, \alpha_{2}, \phi, g\right)$ is called a metric almost contact pair structure on a manifold $M$ and $g$ is an associated metric with respect to contact pair structure $\left(\alpha_{1}, \alpha_{2}, \phi\right)$. We recall $\left(M, \phi, Z_{1}, Z_{2}, \alpha_{1}, \alpha_{2}, g\right)$ is a metric almost contact pair manifold.

We have the following properties for a metric almost contact pair manifold $M$ [16]:

$$
g\left(Z_{i}, Z_{j}\right)=\delta_{i j}, \nabla_{Z_{i}} Z_{j}=0, \nabla_{Z_{i}} \phi=0, \nabla_{X} Z_{1}=-\phi_{1} X, \nabla_{X} Z_{2}=-\phi_{2} X
$$

and for every $X$ tangent to $\mathcal{F}_{i} i=1,2$.

Another major notion for an almost contact manifold is normality. Bande and Hadjar [19] studied on this notion for a metric almost contact pair manifold. They define two almost complex structures on $M$ as [19]:

$$
\mathcal{J}=\phi-\alpha_{2} \otimes Z_{1}+\alpha_{1} \otimes Z_{2}, \quad \mathcal{T}=\phi+\alpha_{2} \otimes Z_{1}-\alpha_{1} \otimes Z_{2} .
$$

Definition 5. A metric almost contact pair manifold is said to be normal if $\mathcal{J}$ and $\mathcal{T}$ are integrable [19]. 
Theorem 1. $\mathcal{J}$ and $\mathcal{T}$ are integrable if the following equation is satisfied;

$$
[\phi, \phi]\left(X_{1}, X_{2}\right)+2 d \alpha_{1}\left(X_{1}, X_{2}\right) Z_{1}+2 d \alpha_{2}\left(X_{1}, X_{2}\right) Z_{2}=0
$$

for all $X_{1}, X_{2} \in \Gamma(T M)$ [19].

From the following theorem, we have the covariant derivation of $\phi$ for a normal metric contact pair manifold

Theorem 2. Let $\left(M, \phi, Z_{1}, Z_{2}, \alpha_{1}, \alpha_{2}, g\right)$ be a normal metric contact pair manifold. Then we have

$$
g\left(\left(\nabla_{X_{1}} \phi\right) X_{2}, X_{3}\right)=\sum_{i=1}^{2}\left(d \alpha_{i}\left(\phi X_{2}, X_{1}\right) \alpha_{i}\left(X_{3}\right)-d \alpha_{i}\left(\phi X_{3}, X_{1}\right) \alpha_{i}\left(X_{2}\right)\right)
$$

for all $X_{1}, X_{2}, X_{3}$ arbitrary vector fields on $M[18]$.

We use the following statements for the Riemann curvature;

$$
R\left(X_{1}, X_{2}\right) X_{3}=\left(\nabla_{X_{1}, X_{2}}^{2}-\nabla_{X_{2}, X_{1}}^{2}\right) X_{3}, \text { where } \nabla_{X_{1}, X_{2}}^{2} X_{3}=\nabla_{X_{1}} \nabla_{X_{2}} X_{3}-\nabla_{\nabla_{X_{1}} X_{2} X_{3}}
$$

for all $X_{1}, X_{2}, X_{3}, X_{4} \in \Gamma(T M)$. Also, it is well known that $R\left(X_{1}, X_{2}, X_{3}, X_{4}\right)=g\left(R\left(X_{1}, X_{2}\right) X_{3}, X_{4}\right)$ [20].

Lemma 1. Let $\left(M, \phi, Z_{1}, Z_{2}, \alpha_{1}, \alpha_{2}, g\right)$ be a normal metric contact pair manifold. Then we have

$$
\begin{gathered}
R\left(X_{1}, Z\right) X_{2}=-g\left(\phi X_{1}, \phi X_{2}\right) Z \\
R\left(X_{1}, X_{2}, Z, X_{3}\right)=\quad \begin{array}{l}
d \alpha_{1}\left(\phi X_{3}, X_{1}\right) \alpha_{1}\left(X_{2}\right)+d \alpha_{2}\left(\phi X_{3}, X_{1}\right) \alpha_{2}\left(X_{2}\right) \\
-d \alpha_{1}\left(\phi X_{3}, X_{2}\right) \alpha_{1}\left(X_{1}\right)-d \alpha_{2}\left(\phi X_{3}, X_{2}\right) \alpha_{2}\left(X_{1}\right), \\
R\left(X_{1}, Z\right) Z=-\phi^{2} X_{1} .
\end{array}
\end{gathered}
$$

where $X_{1}, X_{2}, X_{3} \in \Gamma(T M)$ and $Z=Z_{1}+Z_{2}$ for Reeb vector fields $Z_{1}, Z_{2}$ [21].

Consider an orthonormal basis of $M$ by

$$
S=\left\{e_{1}, e_{2}, \ldots, e_{p}, \phi e_{1}, \phi e_{2}, \ldots, \phi e_{p}, e_{p+1}, e_{p+2}, \ldots, e_{p+q}, \phi e_{p+1}, \phi e_{p+2}, \ldots, \phi e_{p+q}, Z_{1}, Z_{2}\right\} .
$$

Then for all $X_{1} \in \Gamma(T M)$, we get the Ricci curvature of $M$ as

$$
\operatorname{Ric}\left(X_{1}, Z\right)=\sum_{i=1}^{2 p+2 q} d \alpha_{1}\left(\phi E_{i}, E_{i}\right) \alpha_{1}(X)+d \alpha_{2}\left(\phi E_{i}, E_{i}\right) \alpha_{2}(X)
$$

where $E_{i} \in S$.

Lemma 2. Let $\left(M, \phi, Z_{1}, Z_{2}, \alpha_{1}, \alpha_{2}, g\right)$ be a normal metric contact pair manifold. Then Ricci curvature of $M$ satisfies [21]

$$
\begin{gathered}
\operatorname{Ric}\left(X_{1}, Z\right)=0, \text { for } X_{1} \in \Gamma(\mathcal{H}), \\
\operatorname{Ric}(Z, Z)=2 p+2 q . \\
\operatorname{Ric}\left(Z_{1}, Z_{1}\right)=2 p, \operatorname{Ric}\left(Z_{2}, Z_{2}\right)=2 q, \operatorname{Ric}\left(Z_{1}, Z_{2}\right)=0 .
\end{gathered}
$$


In [7], De and Ghosh presented a theorem for the existence of a generalized quasi-Einstein Riemannian manifold.

Theorem 3. A Riemannian manifold is a generalized quasi-Einstein manifold, if the Ricci tensor Ric satisfies the relation

$$
\begin{aligned}
\operatorname{Ric}\left(X_{2}, X_{3}\right) \operatorname{Ric}\left(X_{1}, X_{4}\right)-\operatorname{Ric}\left(X_{1}, X_{3}\right) \operatorname{Ric}\left(X_{2}, X_{4}\right)= & \gamma\left[g\left(X_{2}, X_{3}\right) g\left(X_{1}, X_{4}\right)\right. \\
& \left.-g\left(X_{1}, X_{3}\right) g\left(X_{2}, X_{4}\right)\right]
\end{aligned}
$$

where $\gamma$ is a non-zero scalar [7].

The notion of quasi-constant curvature was defined by Chen and Yano [22]. De and Ghosh generalized this notion for a Riemannian manifold.

Definition 6. Let $M$ be a normal metric contact pair manifold. Then, $M$ is called a normal metric contact pair manifold of generalized quasi-constant curvature if the Riemannian curvature tensor of $M$ satisfying;

$$
\begin{aligned}
R\left(X_{1}, X_{2}, X_{3}, X_{4}\right)= & A\left[g\left(X_{2}, X_{3}\right) g\left(X_{1}, X_{4}\right)-g\left(X_{1}, X_{3}\right) g\left(X_{2}, X_{4}\right)\right] \\
& B\left[g\left(X_{1}, X_{4}\right) \alpha_{1}\left(X_{2}\right) \alpha_{1}\left(X_{3}\right)-g\left(X_{1}, X_{3}\right) \alpha_{1}\left(X_{2}\right) \alpha_{1}\left(X_{4}\right)\right. \\
& \left.+g\left(X_{2}, X_{3}\right) \alpha_{1}\left(X_{1}\right) \alpha_{1}\left(X_{4}\right)-g\left(X_{2}, X_{4}\right) \alpha_{1}\left(X_{1}\right) \alpha_{1}\left(X_{3}\right)\right] \\
& C\left[g\left(X_{1}, X_{4}\right) \alpha_{2}\left(X_{2}\right) \alpha_{2}\left(X_{3}\right)-g\left(X_{1}, X_{3}\right) \alpha_{2}\left(X_{2}\right) \alpha_{2}\left(X_{4}\right)\right. \\
& \left.+g\left(X_{2}, X_{3}\right) \alpha_{2}\left(X_{1}\right) \alpha_{2}\left(X_{4}\right)-g\left(X_{2}, X_{4}\right) \alpha_{2}\left(X_{1}\right) \alpha_{2}\left(X_{3}\right)\right]
\end{aligned}
$$

for all $X_{1}, X_{2}, X_{3}, X_{4} \in \Gamma(T M)$, where $A, B$ and $C$ are scalar functions [7].

Definition 7. Let $M$ be a normal metric contact pair manifold. Then, is called a manifold satisfies cyclic parallel Ricci tensor if we have

$$
\left(\nabla_{X_{1}} \text { Ric }\right)\left(X_{2}, X_{3}\right)+\left(\nabla_{X_{2}} R i c\right)\left(X_{3}, X_{1}\right)+\left(\nabla_{X_{3}} \text { Ric }\right)\left(X_{1}, X_{2}\right)=0
$$

for all $X_{1}, X_{2}, X_{3} \Gamma(T M)[20]$.

Definition 8. Let $M$ be a normal metric contact pair manifold. Then, $M$ is called a manifold satisfies Codazzi type of Ricci tensor if

$$
\left(\nabla_{X_{1}} \operatorname{Ric}\right)\left(X_{2}, X_{3}\right)-\left(\nabla_{X_{2}} R i c\right)\left(X_{1}, X_{3}\right)=0
$$

for all $X_{1} X_{2}$ vector fields on $M[20]$.

Conformal and concircular curvature tensors on contact manifolds have been studied in [23-25]. $\mathcal{M}$-projective curvature tensor on manifolds with different structures studied by many authors [26-28]. These curvature tensors on a normal metric contact pair manifold are defined as below:

Definition 9. Let $M$ be an $(m=2 p+2 q+2)$-dimensional normal metric contact pair manifold. Then,

- $\quad$ M-projective curvature tensor of $M$ is given by [29],

$$
\begin{aligned}
\mathcal{W}\left(X_{1}, X_{2}\right) X_{3}= & R\left(X_{1}, X_{2}\right) X_{3}-\frac{1}{2(m-1)}\left[\operatorname{Ric}\left(X_{2}, X_{3}\right) X_{1}\right. \\
& \left.-\operatorname{Ric}\left(X_{1}, X_{3}\right) X_{2}+g\left(X_{2}, X_{3}\right) Q X_{1}-g\left(X_{1}, X_{3}\right) Q X_{2}\right]
\end{aligned}
$$


- conformal curvature tensor $\mathcal{C}$ of $M$ is given by [20],

$$
\begin{aligned}
\mathcal{C}\left(X_{1}, X_{2}\right) X_{3}= & R\left(X_{1}, X_{2}\right) X_{3} \\
& +\frac{s c a l}{(m-1)(m-2)}\left(g\left(X_{2}, X_{3}\right) X_{1}-g\left(X_{1}, X_{3}\right) X_{2}\right) \\
& +\frac{1}{m-2}\left(g\left(X_{1}, X_{3}\right) Q X_{2}-g\left(X_{2}, X_{3}\right) Q X_{1}\right. \\
& \left.+\operatorname{Ric}\left(X_{1}, X_{3}\right) X_{2}-\operatorname{Ric}\left(X_{2}, X_{3}\right) X_{1}\right)
\end{aligned}
$$

- concircular curvature tensor $\mathcal{Z}$ of $M$ is given by [20],

$$
\mathcal{Z}\left(X_{1}, X_{2}\right) X_{3}=R\left(X_{1}, X_{2}\right) X_{3}-\frac{s c a l}{m(m-1)}\left[g\left(X_{2}, X_{3}\right) X_{1}-g\left(X_{1}, X_{3}\right) X_{2}\right]
$$

for $X_{1}, X_{2}, X_{3} \in \Gamma(T M)$, where $Q$ is Ricci operator is given by $\operatorname{Ric}\left(X_{1}, X_{2}\right)=g\left(Q X_{1}, X_{2}\right)$ and scal is the scalar curvature of $M$.

\section{Generalized Quasi-Einstein Normal Contact Pair Manifolds}

In this section, we present the definition of generalized quasi-Einstein normal metric contact pair manifold. We also present some theorems on the existence and characterizations of generalized quasi-Einstein normal metric contact pair manifold.

Definition 10. Let $M$ be a normal metric contact pair manifold. Then, $M$ is called generalized quasi-Einstein normal metric contact pair manifold if the Ricci curvature of $M$ has the following form;

$$
\operatorname{Ric}\left(X_{1}, X_{2}\right)=\lambda g\left(X_{1}, X_{2}\right)+\beta \alpha_{1}\left(X_{1}\right) \alpha_{1}\left(X_{2}\right)+\mu \alpha_{2}\left(X_{1}\right) \alpha_{2}\left(X_{2}\right)
$$

for functions $\lambda, \beta, \mu$ on $M$ and all $X_{1}, X_{2} \in \Gamma(T M)$.

If we set $X_{1}=X_{2}=Z_{1}$ and $X_{1}=X_{2}=Z_{2}$, respectively, we obtain $\beta=2 p-\lambda$ and $\mu=2 q-\lambda$. Thus, the Ricci curvature of generalized quasi-Einstein normal metric contact pair manifold is given by

$$
\operatorname{Ric}\left(X_{1}, X_{2}\right)=\lambda g\left(X_{1}, X_{2}\right)+(2 p-\lambda) \alpha_{1}\left(X_{1}\right) \alpha_{1}\left(X_{2}\right)+(2 q-\lambda) \alpha_{2}\left(X_{1}\right) \alpha_{2}\left(X_{2}\right)
$$

for all $X_{1}, X_{2} \in \Gamma(T M)$. Therefore, the scalar curvature is

$$
s c a l=2(\lambda+1)(p+q) .
$$

Let $X$ be an arbitrary vector field on $M$. We can write $X=X^{\mathcal{H}}+X^{\mathcal{V}}$. Since the Ricci curvature is a linear tensor we have

$$
\operatorname{Ric}\left(X_{1}, X_{2}\right)=\operatorname{Ric}\left(X_{1}^{\mathcal{H}}, X_{2}^{\mathcal{H}}\right)+\operatorname{Ric}\left(X_{1}^{\mathcal{V}}, X_{2}^{\mathcal{V}}\right) .
$$

Considering the decomposition of tangent bundle mentioned above (see [24] for details), we get

$$
\operatorname{Ric}\left(X_{1}^{\mathcal{V}}, X_{2}^{\mathcal{V}}\right)=2 p \alpha_{1}\left(X_{1}\right) \alpha_{1}\left(X_{2}\right)+2 q \alpha_{2}\left(X_{1}\right) \alpha_{2}\left(X_{2}\right) .
$$

Thus, we reach following useful result.

Proposition 1. A normal metric contact pair manifold is a generalized quasi-Einstein manifold if and only if the horizontal bundle is Einstein, that is for a function $\lambda$ on $M$, we have $\operatorname{Ric}\left(X_{1}^{\mathcal{H}}, X_{2}^{\mathcal{H}}\right)=\lambda g\left(X_{1}^{\mathcal{H}}, X_{2}^{\mathcal{H}}\right)$. 
Assume that (9) is satisfied on a normal metric contact pair manifold $M$. By setting $X_{1}=X_{4}=Z$ and $X_{2}, X_{3} \in \Gamma(\mathcal{H})$, then from (6), we have

$$
\operatorname{Ric}\left(X_{2}, X_{3}\right)=\frac{2 \gamma}{m-2} g\left(X_{2}, X_{3}\right)
$$

Thus, from the Proposition1 $M$ is a generalized quasi-Einstein manifold. Using (13), we get $\gamma=\frac{1}{2}(s c a l-m+2)$ and hence we state;

Corollary 1. Let $M$ be normal metric contact pair manifold with scalar curvature scal $\neq m-2$. If we have the relation

$$
\begin{aligned}
\operatorname{Ric}\left(X_{2}, X_{3}\right) \operatorname{Ric}\left(X_{1}, X_{4}\right)-\operatorname{Ric}\left(X_{1}, X_{3}\right) \operatorname{Ric}\left(X_{2}, X_{4}\right)= & \frac{1}{2}(s c a l-m+2)\left[g\left(X_{2}, X_{3}\right) g\left(X_{1}, X_{4}\right)\right. \\
& \left.-g\left(X_{1}, X_{3}\right) g\left(X_{2}, X_{4}\right)\right]
\end{aligned}
$$

on $M$ for all $X_{1}, X_{2}, X_{3}, X_{4} \in \Gamma(T M)$, then $M$ is a generalized quasi-Einstein manifold.

Let $\pi$ be a plane section in $T_{Q} M$ for any $Q \in M$. The sectional curvature of $\pi$ is given as $\operatorname{Sec}(\pi)=\operatorname{Sec}(u \wedge v)$, where $u, v$ orthonormal vector fields . For any $(p+q)$-dimensional subspace $\mathcal{L} \subset T_{Q} M, 2 \leq p+q \leq m$, its scalar curvature $\operatorname{scal}(\mathcal{L})$ is denoted by

$$
\operatorname{scal}(\mathcal{L})=\sum_{1 \leq i, j \leq p+q} \operatorname{Sec}\left(E_{i} \wedge E_{j}\right)
$$

where $E_{1}, \ldots, E_{n}$ is any orthonormal basis of $\mathcal{L}$ [30]. When $\mathcal{L}=T_{Q} M$, the scalar curvature is just the scalar curvature $\operatorname{scal}(Q)$ of $M$ at $Q \in M$.

The characterizations of Einstein [31,32], quasi-Einstein [33] and generalized quasi-Einstein [11,34] manifolds have been obtained by using the sectional curvature of subspaces of tangent bundle. Analogous to the proof of the Theorem 2.2 of [11], we have following assertion immediately.

Theorem 4. An $(m=2 p+2 q+2)$-dimensional normal metric contact pair manifold is a generalized quasi-Einstein manifold if and only if there exist a function $\lambda$ on $M$ satisfying

$$
\begin{aligned}
\operatorname{scal}(P)+p+q-\lambda & =\sec \left(P^{\perp}\right), Z_{1}, Z_{2} \in T_{Q} P^{\perp} \\
\operatorname{scal}(N)+p+q & =\sec \left(N^{\perp}\right), Z_{1}, Z_{2} \in T_{Q} N^{\perp} \\
\operatorname{scal}(R)+q-p & =\sec \left(P^{\perp}\right), Z_{1} \in T_{Q} R, Z_{2} \in T_{Q} R^{\perp}
\end{aligned}
$$

where $(p+q+1)$-plane sections $P, R$ and $(p+q)$-plane section $N ; P^{\perp}, N^{\perp}$ and $R^{\perp}$ denote the orthogonal complements of $P, N$ and $R$ in $T_{Q} M$, respectively.

We consider the normal metric contact pair manifold is of generalized quasi-constant curvature. In the following proposition, we derive some relations on sectional curvature of $M$.

Proposition 2. Let $M$ be a normal metric contact pair manifold of generalized quasi-constant curvature. Then, we have the following:

- the sectional curvature of horizontal bundle is $A$,

- the sectional curvature of plane section spanned by $X \in \Gamma(\mathcal{H})$ and $Z$ is $2 A+B+C$,

- $\quad$ the sectional curvature of plane section spanned by $X \in \Gamma(\mathcal{H})$ and $Z_{1}, Z_{2}$ is $A+B$ and $A+C$, respectively. 
Proof. Let take $X_{1}=X_{4}=X, X_{2}=X_{4}=X^{\prime}$, where $X, X^{\prime}$ unit and mutually orthogonal horizontal vector fields. Then from (10), we obtain

$$
\begin{aligned}
\sec \left(X, X^{\prime}\right) & =A\left[g\left(X^{\prime}, X^{\prime}\right) g(X, X)-g\left(X, X^{\prime}\right) g\left(X^{\prime}, X\right)\right] \\
& =A
\end{aligned}
$$

For $X_{1}=X_{4}=X, X_{2}=X_{4}=Z$ for unit horizontal vector field $X$, we get

$$
\begin{aligned}
\sec (X, Z)= & A\left[g(Z, Z) g(X, X)-g\left(X, X^{\prime}\right) g\left(X^{\prime}, X\right)-g(X, Z) g(Z, X)\right] \\
& +B\left(g(X, X) \alpha_{1}(Z) \alpha_{1}(Z)+C g(X, X) \alpha_{2}(Z) \alpha_{2}(Z)\right. \\
= & 2 A+B+C
\end{aligned}
$$

Similarly, we can derive the other assertions.

From above proposition, we get

Corollary 2. In a normal metric contact pair manifold of generalized quasi-constant curvature, we have

$$
\sec (X, Z)=\sec \left(X, Z_{1}\right)+\sec \left(X, Z_{2}\right)
$$

for any horizontal and unit vector field $X$.

Theorem 5. A normal metric contact pair manifold of generalized quasi-constant curvature is a generalized quasi-Einstein manifold with coefficients $\lambda=A(m-1)+B+C, \beta=B(m-2)$, and $\mu=C(m-2)$.

Proof. Let $M$ be a normal metric contact pair manifold of generalized quasi-constant curvature. Consider an orthonormal basis of $M$ as

$S=\left\{e_{1}, e_{2}, \ldots, e_{p}, \phi_{1} e_{1}, \phi_{1} e_{2}, \ldots, \phi_{1} e_{p}, e_{p+1}, e_{p+2}, \ldots, e_{p+q}, \phi_{2} e_{p+1}, \phi_{2} e_{p+2}, \ldots, \phi_{2} e_{p+q}, Z_{1}, Z_{2}\right\}$.

By taking sum of (10) from $i=1$ to $i=2 p+2 q+2$ for $X_{2}=X_{3}=E_{i} \in S$, we obtain

$$
\begin{aligned}
\sum_{i=1}^{2 p+2 q+2} R\left(X_{1}, E_{i}, E_{i}, X_{4}\right)= & \sum_{i=1}^{2 p+2 q+2}\left\{A\left[g\left(E_{i}, E_{i}\right) g\left(X_{1}, X_{4}\right)-g\left(X_{1}, E_{i}\right) g\left(E_{i}, X_{4}\right)\right]\right. \\
& B\left[g\left(X_{1}, X_{4}\right) \alpha_{1}\left(E_{i}\right) \alpha_{1}\left(E_{i}\right)-g\left(X_{1}, E_{i}\right) \alpha_{1}\left(E_{i}\right) \alpha_{1}\left(X_{4}\right)\right. \\
& \left.+g\left(E_{i}, E_{i}\right) \alpha_{1}\left(X_{1}\right) \alpha_{1}\left(X_{4}\right)-g\left(E_{i}, X_{4}\right) \alpha_{1}\left(X_{1}\right) \alpha_{1}\left(E_{i}\right)\right] \\
& C\left[g\left(X_{1}, X_{4}\right) \alpha_{2}\left(E_{i}\right) \alpha_{2}\left(E_{i}\right)-g\left(X_{1}, E_{i}\right) \alpha_{2}\left(E_{i}\right) \alpha_{2}\left(X_{4}\right)\right. \\
& \left.\left.+g\left(E_{i}, E_{i}\right) \alpha_{2}\left(X_{1}\right) \alpha_{2}\left(X_{4}\right)-g\left(E_{i}, X_{4}\right) \alpha_{2}\left(X_{1}\right) \alpha_{2}\left(E_{i}\right)\right]\right\} .
\end{aligned}
$$

For $1 \leq i \leq 2 p+2 q$ since $\alpha_{j}\left(E_{i}\right)=0, j=1,2$ and $\sum_{i=1}^{2 p+q+2} g\left(X_{1}, E_{i}\right) g\left(E_{i}, X_{2}\right)=g\left(X_{1}, X_{2}\right)$ we get

$$
\begin{aligned}
\operatorname{Ric}\left(X_{1}, X_{4}\right)= & {[A(m-1)+B+C] g\left(X_{1}, X_{4}\right)+B(m-2) \alpha_{1}\left(X_{1}\right) \alpha_{1}\left(X_{4}\right) } \\
& +C(m-2) \alpha_{2}\left(X_{1}\right) \alpha_{2}\left(X_{4}\right)
\end{aligned}
$$

which completes the proof.

\section{Normal Metric Contact Pair Manifold Satisfying Certain Conditions on Ricci Tensor}

De and Mallick[9] proved that a generalized quasi-Einstein Riemann manifold satisfies cyclic parallel Ricci tensor if generators of the manifolds are Killing vector fields. As we know that the 
characteristic vector fields of a normal metric contact pair manifold $Z_{1}, Z_{2}$ are Killing vector fields [35]. Thus, by easy computations, we get

$$
\left(\nabla_{X_{1}} \alpha_{1}\right) X_{2}+\left(\nabla_{X_{2}} \alpha_{1}\right) X_{1}=0,\left(\nabla_{X_{1}} \alpha_{2}\right) X_{2}+\left(\nabla_{X_{2}} \alpha_{2}\right) X_{1}=0
$$

for all $X_{1}, X_{2} \in \Gamma(T M)$. On the other hand, we have

$$
\left(\nabla_{X_{1}} \operatorname{Ric}\right)\left(X_{2}, X_{3}\right)=\nabla_{X_{1}} \operatorname{Ric}\left(X_{2}, X_{3}\right)-\operatorname{Ric}\left(\nabla_{X_{1}} X_{2}, X_{3}\right)-\operatorname{Ric}\left(X_{2}, \nabla_{X_{1}} X_{3}\right)
$$

for all $X_{1}, X_{2}, X_{3} \in \Gamma(T M)$ [20]. Then, from (12) we obtain

$$
\begin{aligned}
\left(\nabla_{X_{1}} R i c\right)\left(X_{2}, X_{3}\right)= & X_{1}[\lambda] g\left(\phi X_{2}, \phi X_{3}\right) \\
& +(2 p-\lambda)\left[\left(\left(\nabla_{X_{1}} \alpha_{1}\right) X_{3}\right) \alpha_{1}\left(X_{3}\right)+\left(\left(\nabla_{X_{1}} \alpha_{1}\right) X_{2}\right) \alpha_{1}\left(X_{2}\right)\right] \\
& (2 q-\lambda)\left[\left(\left(\nabla_{X_{1}} \alpha_{1}\right) X_{3}\right) \alpha_{2}\left(X_{2}\right)+\left(\left(\nabla_{X_{1}} \alpha_{1}\right) X_{2}\right) \alpha_{2}\left(X_{3}\right)\right]
\end{aligned}
$$

where $X_{1}[\lambda]$ is the derivation of $\lambda$ in the direction of $X_{1}$. Thus, from (15), we obtain

$$
\begin{aligned}
& \left(\nabla_{X_{1}} R i c\right)\left(X_{2}, X_{3}\right)+\left(\nabla_{X_{2}} R i c\right)\left(X_{3}, X_{1}\right)+\left(\nabla_{X_{3}} R i c\right)\left(X_{1}, X_{2}\right) \\
= & X_{1}[\lambda] g\left(\phi X_{2}, \phi X_{3}\right)+X_{2}[\lambda] g\left(\phi X_{3}, \phi X_{1}\right)+X_{3}[\lambda] g\left(\phi X_{1}, \phi X_{2}\right) .
\end{aligned}
$$

As a consequence, we can state the following theorem.

Theorem 6. Let $M$ be a generalized quasi-Einstein normal metric contact pair manifold. If $\lambda$ is constant then $M$ satisfies cyclic parallel Ricci tensor.

In [9], it has been proved that if a generalized quasi-Einstein Riemann manifold satisfies Codazzi type of Ricci tensor, then the associated 1-forms are closed.

Suppose that Ricci tensor Ric of a normal metric contact pair manifold $M$ is Codazzi type. Then, from (15) and (16) we obtain

$$
\begin{aligned}
& (2 p-\lambda)\left[\left(\left(\nabla_{X_{1}} \alpha_{1}\right) X_{2}-\left(\nabla_{X_{2}} \alpha_{1}\right) X_{1}\right) \alpha_{1}\left(X_{3}\right)\right. \\
& \left.+\left(\left(\nabla_{X_{1}} \alpha_{1}\right) X_{3} \alpha_{1}\left(X_{2}\right)+\left(\nabla_{X_{2}} \alpha_{1}\right) X_{3} \alpha_{1}\left(X_{1}\right)\right)\right] \\
& +(2 q-\lambda)\left[\left(\left(\nabla_{X_{1}} \alpha_{1}\right) X_{2}-\left(\nabla_{X_{2}} \alpha_{1}\right) X_{1}\right) \alpha_{2}\left(X_{3}\right)\right. \\
& \left.+\left(\left(\nabla_{X_{1}} \alpha_{1}\right) X_{3} \alpha_{2}\left(X_{2}\right)+\left(\nabla_{X_{2}} \alpha_{1}\right) X_{3} \alpha_{2}\left(X_{1}\right)\right)\right]=0 .
\end{aligned}
$$

Let take $X_{3}=Z_{1}$, then we get

$$
(2 p-\lambda)\left(\left(\nabla_{X_{1}} \alpha_{1}\right) X_{2}-\left(\nabla_{X_{2}} \alpha_{1}\right) X_{1}\right)=0
$$

which implies $\lambda=2 p$ or $\left(\nabla_{X_{1}} \alpha_{1}\right) X_{2}-\left(\nabla_{X_{2}} \alpha_{1}\right) X_{1}=0$. If $\lambda=2 p$ then the manifold is not generalized quasi-Einstein, so this case is not possible. In the other case we obtain

$$
0=\left(\nabla_{X_{1}} \alpha_{1}\right) X_{2}-\left(\nabla_{X_{2}} \alpha_{1}\right) X_{1}=d \alpha_{1}\left(X_{1}, X_{2}\right)=0
$$

and so $\alpha_{1}$ is closed. Similarly, by choosing $X_{3}=Z_{2}$ we obtain $\alpha_{2}$ is closed. As we know contact pairs $\left(\alpha_{1}, \alpha_{2}\right)$ are not closed. So, our assumption is not valid. Finally, we conclude that

Theorem 7. A generalized quasi-Einstein normal metric contact pair manifold does not satisfy Codazzi type of Ricci tensor. 


\section{Normal Metric Contact Pair Manifold Satisfying Certain Curvature Conditions}

Curvature tensors give us many geometric properties of contact manifolds. Some properties of normal metric contact pair manifold satisfying certain conditions of curvature tensors were given in $[21,24]$. In this section, we examine the $\mathcal{M}$-projective curvature tensor $\mathcal{W}$, conformal curvature tensor $\mathcal{C}$ and concircular curvature tensor $\mathcal{Z}$ on a normal metric contact pair manifold.

From (11), we have

$$
\begin{aligned}
\mathcal{W}\left(X_{1}, Z\right) Z & =\frac{m}{2 m-1} X_{1}-\frac{1}{m-1} Q X_{1} \\
\mathcal{W}\left(X_{1}, X_{2}\right) Z & =R\left(X_{1}, X_{2}\right) Z \\
\mathcal{W}\left(X_{1}, Z\right) X_{2} & =\left[\frac{(2 m-1)(m-2)}{2(m-1)} g\left(X_{1}, X_{2}\right)+\frac{1}{2(m-1)} \operatorname{Ric}\left(X_{1}, X_{2}\right)\right] Z
\end{aligned}
$$

for $X_{1}, X_{2}, X_{3} \in \Gamma(\mathcal{H})$. Also, since $\operatorname{Ric}\left(X_{1}, X_{2}\right)=g\left(Q X_{1}, X_{2}\right)$, where $Q$ is the Ricci operator, we have

$$
\begin{aligned}
\mathcal{W}\left(X_{1}, X_{2}, X_{3}, X_{4}\right) & =R\left(X_{1}, X_{2}, X_{3}, X_{4}\right)-\frac{1}{2(m-1)}\left[\operatorname{Ric}\left(X_{2}, X_{3}\right) g\left(X_{1}, X_{4}\right)\right. \\
& -\operatorname{Ric}\left(X_{1}, X_{3}\right) g\left(X_{2}, X_{4}\right)+g\left(X_{2}, X_{3}\right) \operatorname{Ric}\left(X_{1}, X_{4}\right) \\
& \left.-g\left(X_{1}, X_{3}\right) \operatorname{Ric}\left(X_{2}, X_{4}\right)\right] .
\end{aligned}
$$

for all $X_{1}, X_{2}, X_{3} \in \Gamma(M) . M$ is called $\mathcal{M}$-projectively flat if $\mathcal{W}$ vanishes identically on $M$.

Theorem 8. A generalized quasi-Einstein normal metric contact pair manifold is $\mathcal{M}$-projectively flat if and only if it is of generalized quasi-constant curvature.

Proof. Suppose that $M$ is a generalized quasi-Einstein manifold. Then, from (12) and (11) we have

$$
\begin{aligned}
\mathcal{W}\left(X_{1}, X_{2}, X_{3}, X_{4}\right)= & R\left(X_{1}, X_{2}, X_{3}, X_{4}\right) \\
& -\frac{\lambda}{m-1}\left[g\left(X_{2}, X_{3}\right) g\left(X_{1}, X_{4}\right)-g\left(X_{1}, X_{3}\right) g\left(X_{2}, X_{4}\right)\right] \\
& -\frac{2 p-\lambda}{2(m-1)}\left[g\left(X_{1}, X_{4}\right) \alpha_{1}\left(X_{2}\right) \alpha_{1}\left(X_{3}\right)-g\left(X_{1}, X_{3}\right) \alpha_{1}\left(X_{2}\right) \alpha_{1}\left(X_{4}\right)\right. \\
& \left.+g\left(X_{2}, X_{3}\right) \alpha_{1}\left(X_{1}\right) \alpha_{1}\left(X_{4}\right)-g\left(X_{2}, X_{4}\right) \alpha_{1}\left(X_{1}\right) \alpha_{1}\left(X_{3}\right)\right] \\
& -\frac{2 q-\lambda}{2(m-1)}\left[g\left(X_{1}, X_{4}\right) \alpha_{2}\left(X_{2}\right) \alpha_{2}\left(X_{3}\right)-g\left(X_{1}, X_{3}\right) \alpha_{2}\left(X_{2}\right) \alpha_{2}\left(X_{4}\right)\right. \\
& \left.+g\left(X_{2}, X_{3}\right) \alpha_{2}\left(X_{1}\right) \alpha_{2}\left(X_{4}\right)-g\left(X_{2}, X_{4}\right) \alpha_{2}\left(X_{1}\right) \alpha_{2}\left(X_{3}\right)\right] .
\end{aligned}
$$

Thus, it is seen that $M$ is $\mathcal{M}$-projectively flat if and only if $M$ is of generalized quasi-constant curvature with coefficients $A=\frac{\lambda}{m-1}, B=\frac{2 p-\lambda}{2(m-1)}$ and $C=\frac{2 q-\lambda}{2(m-1)}$.

The Riemann manifolds satisfying $R\left(X_{1}, X_{2}\right) \cdot R=0$ are called semi-symmetric, where $R\left(X_{1}, X_{2}\right)$ acts on $R$ as a derivation. Semi-symmetric contact manifolds were studied by Perrone [36]. Similarly, if $\mathcal{W}\left(X_{1}, X_{2}\right) \cdot R=0$ then $M$ is called $\mathcal{M}$-projectively semi-symmetric. $\mathcal{W}\left(X_{1}, X_{2}\right) \cdot R$ is defined as

$$
\begin{aligned}
\left(\mathcal{W}\left(X_{1}, X_{2}\right) \cdot R\right)\left(X_{3}, X_{4}\right) X_{5} & =\mathcal{W}\left(X_{1}, X_{2}\right) \cdot R\left(X_{3}, X_{4}\right) X_{5}-R\left(\mathcal{W}\left(X_{1}, X_{2}\right), X_{3}, X_{4}\right) X_{5} \\
& -R\left(X_{3}, \mathcal{W}\left(X_{1}, X_{2}\right) X_{4}\right) X_{5}-R\left(X_{3}, X_{4}\right) \mathcal{W}\left(X_{1}, X_{2}\right) X_{5}
\end{aligned}
$$

for all $X_{1}, X_{2}, X_{3}, X_{4}, X_{5} \in \Gamma(T M)$. Also, we have

$$
\left(\mathcal{W}\left(X_{1}, X_{2}\right) \cdot \operatorname{Ric}\right)\left(X_{3}, X_{4}\right)=-\operatorname{Ric}\left(\mathcal{W}\left(X_{1}, X_{2}\right), X_{3}\right)-\operatorname{Ric}\left(X_{3}, \mathcal{W}\left(X_{1}, X_{2}\right) X_{4}\right) .
$$

If $\mathcal{W}\left(X_{1}, X_{2}\right) \cdot$ Ric $=0$ then $M$ is called $\mathcal{M}$-projectively Ricci semi-symmetric.

Theorem 9. A normal metric contact pair manifold is $\mathcal{M}$-projectively semi-symmetric if and only if $M$ is a generalized quasi-Einstein manifold. 
Proof. From (22) and using (17)-(19) we obtain

$$
\begin{aligned}
\left(\mathcal{W}\left(X_{1}, Z\right) \cdot R\right)\left(X_{3}, X_{4}\right) X_{5}= & K R\left(X_{1}, X_{3}, X_{4}, X_{5}\right) Z+L R\left(X_{3}, X_{4}, X_{5}, Q X_{1}\right) Z \\
& -\left(K g\left(X_{1}, X_{3}\right)+L R i c\left(X_{1}, X_{3}\right)\right) g\left(X_{4}, X_{5}\right) Z \\
& +\left(K g\left(X_{1}, X_{5}\right)+\operatorname{LRic}\left(X_{1}, X_{5}\right)\right) R\left(X_{3}, X_{4}\right) Z
\end{aligned}
$$

where $K=\frac{(2 m-1)(m-2)}{2(m-1)}$ and $L=\frac{1}{2(m-1)}$.

Let take $X_{1}, X_{3}, X_{5}$ horizontal vector fields and $X_{4}=Z$, from (3)-(7), we get

$$
\left(\mathcal{W}\left(X_{1}, Z\right) \cdot R\right)\left(X_{3}, Z\right) X_{5}=-\left(K g\left(X_{1}, X_{5}\right)+\operatorname{LRic}\left(X_{1}, X_{5}\right)\right) X_{3}
$$

Thus, we conclude that $\left(\mathcal{W}\left(X_{1}, Z\right) \cdot R\right)\left(X_{3}, X_{4}\right) X_{5}=0$ if and only if horizontal bundle of $M$ is Einstein. From Proposition 1, we obtain

$$
\operatorname{Ric}\left(X_{1}, X_{5}\right)=-\frac{K}{L} g\left(X_{1}, X_{5}\right)+\left(2 p+\frac{K}{L}\right) \alpha_{1}\left(X_{1}\right) \alpha_{1}\left(X_{5}\right)+\left(2 q+\frac{K}{L}\right) \alpha_{2}\left(X_{1}\right) \alpha_{2}\left(X_{5}\right)
$$

Therefore, the manifold is a generalized quasi-Einstein.

Theorem 10. An $(m=2 p+2 q+2)$-dimensional normal metric contact pair manifold satisfies $\mathcal{W} \cdot R i c=0$ if and only $M$ is generalized quasi-Einstein manifold.

Proof. For $X_{1}, X_{3}, X_{4} \in \Gamma(T M)$ from (23) we get

$$
\begin{aligned}
\left(\mathcal{W}\left(X_{1}, Z\right) \cdot \operatorname{Ric}\right)\left(X_{3}, X_{4}\right)= & -\operatorname{Ric}\left(\left[\operatorname{Kg}\left(X_{1}, X_{3}\right)+\operatorname{LRic}\left(X_{1}, X_{3}\right)\right] Z, X_{4}\right) \\
& -\operatorname{Ric}\left(X_{3},\left[\operatorname{Kg}\left(X_{1}, X_{4}\right)+\operatorname{LRic}\left(X_{1}, X_{4}\right)\right] Z\right) .
\end{aligned}
$$

Let take $X_{1}, X_{4}$ vector fields and $X_{3}=Z$ from (6), (7), we obtain

$$
\left(\mathcal{W}\left(X_{1}, Z\right) \cdot \operatorname{Ric}\right)\left(X_{3}, X_{4}\right)=-(2 p+2 q)\left[K g\left(X_{1}, X_{4}\right)+\operatorname{LRic}\left(X_{1}, X_{4}\right)\right]
$$

Therefore, $\left(\mathcal{W}\left(X_{1}, Z\right) \cdot R i c\right)\left(X_{3}, X_{4}\right)=0$ if and only if horizontal bundle is Einstein. From Proposition (1) we get (24), which completes the proof.

Blair, Bande and Hadjar [21] studied on conformal flatness of normal metric contact pair manifolds and they proved following theorem.

Theorem 11. A conformally flat normal metric contact pair manifold is locally isometric to the Hopf manifold $S^{2 q+1}(1) \times S^{1}[21]$.

Thus, we get following results, for a generalized quasi-Einstein normal metric contact pair manifold.

Theorem 12. Let $M$ be a generalized quasi-Einstein normal metric contact pair manifold. If $M$ is of generalized quasi-constant curvature with coefficients $A=\frac{\lambda m-m+2}{(m-1)(m-2)}, B=\frac{2 p-\lambda}{m-2}$ and $C=\frac{2 q-\lambda}{m-2}$, then it is locally isometric to the Hopf manifold $S^{2 q+1}(1) \times S^{1}$. 
Proof. Let $M$ be a generalized quasi-Einstein normal metric contact pair manifold. Then, we have

$$
\begin{aligned}
\mathcal{C}\left(X_{1}, X_{2}, X_{3}, X_{4}\right)= & R\left(X_{1}, X_{2}, X_{3}, X_{4}\right) \\
& +\frac{s c a l}{(m-1)(m-2)}\left[g\left(X_{2}, X_{3}\right) g\left(X_{1}, X_{4}\right)-g\left(X_{1}, X_{3}\right) g\left(X_{2}, X_{4}\right)\right] \\
& -\frac{2 \lambda}{m-2}\left[g\left(X_{2}, X_{3}\right) g\left(X_{1}, X_{4}\right)-g\left(X_{1}, X_{3}\right) g\left(X_{2}, X_{4}\right)\right] \\
& -\frac{2 p-\lambda}{m-2}\left[g\left(X_{1}, X_{4}\right) \alpha_{1}\left(X_{2}\right) \alpha_{1}\left(X_{3}\right)-g\left(X_{1}, X_{3}\right) \alpha_{1}\left(X_{2}\right) \alpha_{1}\left(X_{4}\right)\right. \\
& \left.+g\left(X_{2}, X_{3}\right) \alpha_{1}\left(X_{1}\right) \alpha_{1}\left(X_{4}\right)-g\left(X_{2}, X_{4}\right) \alpha_{1}\left(X_{1}\right) \alpha_{1}\left(X_{3}\right)\right] \\
& -\frac{2 q-\lambda}{m-2}\left[g\left(X_{1}, X_{4}\right) \alpha_{2}\left(X_{2}\right) \alpha_{2}\left(X_{3}\right)-g\left(X_{1}, X_{3}\right) \alpha_{2}\left(X_{2}\right) \alpha_{2}\left(X_{4}\right)\right. \\
& \left.-g\left(X_{2}, X_{4}\right) \alpha_{2}\left(X_{1}\right) \alpha_{2}\left(X_{3}\right)\right] .
\end{aligned}
$$

Suppose that $M$ is of generalized quasi-constant curvature with coefficients $A=\frac{\lambda m-m+2}{(m-1)(m-2)}, B=$ $\frac{2 p-\lambda}{m-2}$ and $C=\frac{2 q-\lambda}{m-2}$. Then, we get $\mathcal{C}=0$ which means that $M$ is conformally flat. Thus, the Theorem 6.4 $M$ is locally isometric to the Hopf manifold $S^{2 q+1}(1) \times S^{1}$.

By using the definition of $\mathcal{M}$-projective curvature tensor and conformal curvature tensor, we have

$$
\begin{aligned}
\mathcal{C}\left(X_{1}, X_{2}\right) X_{3}= & \frac{2(m-1)}{m-2} \mathcal{W}\left(X_{1}, X_{2}\right) X_{3}-\frac{m}{m-2} R\left(X_{1}, X_{2},\right) X_{3} \\
& +\frac{s c a l}{(m-1)(m-2)}\left[g\left(X_{2}, X_{3}\right) X_{1}-g\left(X_{1}, X_{3}\right) X_{2}\right]
\end{aligned}
$$

Let $M$ be a $\mathcal{M}$-projectively flat normal metric contact pair manifold, then, from (25), $M$ is conformally flat if and only if

$$
R\left(X_{1}, X_{2},\right) X_{3}=\frac{s c a l}{m(m-1)}\left[g\left(X_{2}, X_{3}\right) X_{1}-g\left(X_{1}, X_{3}\right) X_{2}\right],
$$

which means $M$ is concircular flat. Finally, we conclude that

Theorem 13. Let $M$ be $\mathcal{M}$-projectively flat normal metric contact pair manifold. If $M$ is also concircularly flat then it is locally isometric to Hopf manifold $S^{2 q+1}(1) \times S^{1}$.

Funding: This research received no external funding.

Acknowledgments: The author would like to thank Uday CHAND DE and Aysel TURGUT VANLI for their helpful suggestions and their valuable comments which helped to improve the manuscript.

Conflicts of Interest: The authors declare no conflict of interest.

\section{References}

1. Chaki, M.C. On quasi Einstein manifolds. Publ. Math. 2000, 57, 297-306.

2. De, U.C.; Ghosh, G.C. On quasi Einstein manifolds. Periodica Mathematica Hungarica 2004, 48, $223-231$. [CrossRef]

3. Ghosh, G.C.; De, U.C.; Binh, T.Q. Certain curvature restrictions on a quasi Einstein manifolds. Publ. Math. Debr. 2006, 69, 209-217.

4. Tripathi, M.M.; Kim, J. On N(k)-quasi Einstein manifolds. Commun. Korean Math. Soc. 2007, $22,411$. [CrossRef]

5. Chaki, M.C. On Generalized quasi-Einstein manifold. Publ. Math. Debrecen 2001, 58, 638-691.

6. Catino, G. Generalized quasi-Einstein manifolds with harmonic Weyl tensor. Mathematische Zeitschrift 2012, 271,751-756. [CrossRef]

7. De, U.C.; Ghosh, G.C. On generalized quasi Einstein manifolds. Kyungpook Math. J. 2004, 44, 607. [CrossRef]

8. De, U.C.; Mallick, S. On the existence of generalized quasi-Einstein manifolds. Arch. Math. 2011, 47, $279-291$. 
9. De, U.C.; Mallick, S. On generalized quasi-Einstein manifolds admitting certain vector fields. Filomat 2015, 29, 599-609. [CrossRef]

10. Prakasha, D.G.; Venkatesha, H. Some results on generalized quasi-Einstein manifolds. Chin. J. Math. 2014, 18, 563803 [CrossRef]

11. Sular, S.; Özgür, C. Characterizations of generalized quasi-Einstein manifolds. An. Univ. Ovidius Constanta-Seria Mat. 2012, 20, 407-416. [CrossRef]

12. De, U.C.; Shenawy, S. Generalized quasi-Einstein GRW space-times. Int. J. Geom. Methods Mod. Phys. 2019, 16, 1950124. [CrossRef]

13. Güler, S.; Demirbağ, S.A. A study of generalized quasi Einstein spacetimes with applications in general relativity. Int. J. Theor. Phys. 2016, 55 548-562. [CrossRef]

14. Mallick, S.; De, U.C. On a Class of Generalized quasi-Einstein Manifolds with applications to relativity. Acta Univ. Palacki. Olomuc. Fac. Rerum Nat. Math. 2016, 55, 111-127.

15. Turgut Vanlı, A.; Ünal, İ. On complex $\eta$-Einstein normal complex contact metric manifolds. Commun. Math. Appl. 2017, 8, 301-313.

16. Bande, G.; Hadjar, A. Contact pairs. Tohoku Math. J. 2005, 57, 247-260. [CrossRef]

17. Blair, D.E.; Ludden, G.D.; Yano, K. Geometry of complex manifolds similar to the Calabi-Eckmann manifolds. J. Differ. Geom. 1974, 9, 263-274. [CrossRef]

18. Bande, G.; Hadjar, A. Contact pair structures and associated metrics. Differ. Geom.-Proc. VIII Int. Colloq. 2009, 266-275. [CrossRef]

19. Bande, G.; Hadjar, A. On normal contact pairs. Int. J. Math. 2010, 21, 266-275. [CrossRef]

20. Yano, K.; Kon, M. Structures on Manifolds. World Sci. 1985. [CrossRef]

21. Bande, G.; Blair, D.E.; Hadja, A. Bochner and conformal flatness of normal metric contact pairs. Ann. Glob. Anal. Geom. 2015, 48, 47-56. [CrossRef]

22. Chen, B.Y.; Yano, K. Hypersurfaces of a conformally flat space. Tensor (NS) 1972, 26, 318-322.

23. Unal, İ.; Sarı, R.; Turgut Vanlı, A. Concircular curvature tensor on generalized kenmotsu manifolds. Gümüşhane Üniversitesi Fen Bilimleri Enstitüsü Dergisi 2018, CMES 2018, 99-105.

24. Ünal, İ. Some flatness conditions on normal metric contact pairs. Commun. Fac. Sci. Univ. Ank. Ser. A1 Math. Stat. 2020, 69, 1-10

25. Turgut Vanlı, A.; Ünal, İ. Conformal, concircular, quasi-conformal and conharmonic flatness on normal complex contact metric manifolds. Int. J. Geom. Methods Mod. Phys. 2017, 14, 1750067. [CrossRef]

26. Prakasha D.G.; Fernandez, L.M.; Mirji, K. The $\mathcal{M}$-projective curvature tensor field on generalized $(\kappa, \mu)$-paracontact metric manifolds. Georgian Math. J. 2020, 27, 141-147. [CrossRef]

27. Ayar, G.; Chaubey, S.K. M-projective curvature tensor over cosymplectic manifolds. Differ. Geom. Dyn. Syst. 2019, 21, 23-33.

28. Zengin, F.Ö. On m-projectively flat LP-Sasakian manifolds. Ukrainian Math. J. 2014, 65, 1725-1732. [CrossRef]

29. Pokhariyal, G.P.; Mishra, R.S. Curvature tensors and their relativistics significance. Yokohama Math. J. 1970, 18, 105-108.

30. Chen, B.Y. Some new obstructions to minimal and Lagrangian isometric immersions. Jpn. J. Math. 2000, 26, 105-127. [CrossRef]

31. Chen, B.Y.; Dillen, F.; Verstraelen, L.; Vrancken, L. Characterizations of Riemannian space forms, Einstein spaces and conformally flat spaces. Proc. Am. Math. Soc. 2000, 128, 589-598. [CrossRef]

32. Dumitru, D. On Einstein spaces of odd dimension. Bul. Univ. Transilvania Brasov. 2007, 14, 95-97.

33. Bande, G.; Blair, D.E.; Hadja, A. Characterization of quasi-Einstein manifolds. An. Stiint. Univ. AL. I. Cuza Iasi. Mat. (NS) 2007, 53, 67-72.

34. Dumitru, D. A characterization of generalized quasi-Einstein manifolds. Novi Sad J. Math. 2012, 142, 89-94.

35. Bande, G.; Hadjar, A. Symmetry in the geometry of metric contact pairs. Mathematische Nachrichten 2013, 286, 1701-1709. [CrossRef]

36. Perrone, D. Contact Riemannian manifolds satisfying $R(X, \xi) R=0$. Yokohama Math. J. 1992, 39, 141-149.

(C) 2020 by the authors. Licensee MDPI, Basel, Switzerland. This article is an open access article distributed under the terms and conditions of the Creative Commons Attribution (CC BY) license (http:/ / creativecommons.org/licenses/by/4.0/). 